\title{
Block correlation and the spatial resolution of soil property maps made by kriging.
}

\author{
R.M. Lark* \\ British Geological Survey, Keyworth, Nottinghamshire NG12 5GG, U.K.
}

\begin{abstract}
1 The block correlation is the correlation between the block kriging prediction of a variable and the true spatial mean which it estimates, computed for a particular sampling configuration and block size over the stochastic model which underlies the kriging prediction. This

4 correlation can be computed if the variogram and disposition of sample points are known. It

5 is also possible to compute the concordance correlation, a modified correlation which mea-

6 sures the extent to which the block kriging prediction and true block spatial mean conform

7 to the 1:1 line, and so is sensitive to the tendency of the kriging predictor to over-smooth.

8 It is proposed that block concordance correlation has two particular advantages over krig-

9 ing variance for communicating uncertainty in predicted values. First, as a measure on a bounded scale it is more intuitively understood by the non-specialist data user, particularly one who is interested in a synoptic overview of soil variation across a region. Second, because it accounts for the variability of the spatial means and their kriged estimates, as well as the uncertainty of the latter, it can be more readily compared between blocks of different size than can a kriging variance.

Using the block correlation and concordance correlation it is shown that the uncertainty of block kriged predictions depends on block size, but this effect depends on the interaction of the autocorrelation of the random variable and the sampling intensity. In some circumstances (where the dominant component of variation is at a long range relative to sample spacing) the block correlation and concordance correlation are insensitive to block size, but if the grid spacing is closer to the range of correlation of a significant component then block size can have a substantial effect on block correlation. It is proposed that (i) block concordance correlation is used to communicate the uncertainty in kriged predictions to a range of audiences (ii) that it is used to explore sensitivity to block size when planning mapping and (iii) as a general operational rule a block size is selected to give a block concordance correlation of 0.8 or larger where this can be achieved without extra sampling. Keywords: Kriging, Quality measures, Spatial resolution.
\end{abstract}

${ }^{*}$ E-mail address: mlark@nerc.ac.uk (R.M. Lark). 
In conventional soil survey the scale of the published map plays a tacit role in communicating to the user an understanding of the uncertainty in the information that it conveys.

30 The intensity of field effort affects the uncertainty of predictions made from the resulting soil

31 map in terms of soil classes and soil properties (Beckett and Burrough, 1971). Soil survey

32 organizations have conventionally required that maps be supported by some fixed density

33 of field observations on the published map (Burrough and Beckett, 1971). For example, soil

34 maps in British Columbia should be supported by about 1 observation per $\mathrm{cm}^{2}$ of published

35 map, with an acceptable range of $0.2-2$ observations $\mathrm{cm}^{-2}$ (Resources Inventory Committee,

${ }_{36}$ 1995). In Australia it has been recommended that the density of observations is in the range

${ }_{37} \quad 0.25-1 \mathrm{~cm}^{-2}$ (Gunn et al, 1988). The larger the density of observations on the ground the

38 larger the cartographic scale of the map which these observations can support. For this

39 reason, the larger the scale ratio of a map the greater confidence a user can have in predict-

40 ing likely soil conditions at a site. Maps published at different scales are therefore suitable

${ }_{41}$ for different purposes. Large scale maps (e.g. 1:10000-1:25000) are called 'detailed' maps

42 and are recommended for agricultural extension and irrigation planning, whereas maps at

43 1:120000 - 1:500000 are called 'reconnaissance' maps and are recommended for national-

44 scale land use planning and tentative selection of project locations (Dent and Young, 1981).

45 The printed map with a particular cartographic scale ratio is increasingly superseded

${ }_{46}$ by the raster layer in a Geographical Information System (GIS), a digital soil map. As

${ }_{47}$ GIS technology emerged cartographers explored how their traditional questions about scale

48 ratios should be addressed in this new setting. They made a link with the spatial resolution,

49 expressed by the dimension of the raster cell or pixel (I assume square pixels in this paper).

so Tobler (1988) proposed a heuristic rule by which information that could be communicated

${ }_{51}$ effectively on a map of scale ratio no smaller than $1: s$ requires raster cells length $r$ such that

${ }_{52} \quad r \leq s / 2000 \mathrm{~m}$. Hengl (2006) reviewed and proposed a similar set of rules, for example, if the 
53 effective range of the variogram of a continuous variable is $a$ units then this variable should

${ }_{54}$ be mapped on pixels of length $r \leq a / 2$.

${ }_{55}$ The question of the resolution of a raster soil map, here I focus on maps of individual 56 soil properties, has received revived attention with the emergence of the GlobalSoilMap ${ }_{57}$ project (Hempel et al., 2014). The project specification (Arrouays et al., 2014a) requires ${ }_{58}$ that, in addition to a grid of predictions on a notional $2 \times 2-\mathrm{m}$ 'pedon' support (first tier 59 information), the spatial mean of soil properties, to a specified depth, is predicted over a $60100 \times 100-m$ block (second tier information).

As Arrouays et al. (2014b) acknowledge, this selection of a cell size was not un-

62 controversial. In particular they note that the data user is likely make inferences about

63 uncertainty from block size (resolution), and that a map of global extent on 100-m blocks,

${ }_{64}$ given available data, may seem implausibly ambitious. They observe, however, that in the

65 context of digital soil mapping the uncertainty of an individual prediction, on a block of any

${ }_{66}$ size, can be quantified directly. Uncertainty, therefore, should not be tacitly communicated

${ }_{67}$ by scale or block size, and one is free to select a block size on the basis of other criteria such

${ }_{68}$ as the resolution of ancillary data to be used in the prediction.

69 This argument is correct, but it is necessary to reflect on how this approach adds to the challenge of successfully communicating the uncertainty attached to soil information to

71 the user of this information. Three points are particularly germane.

${ }_{72}$ First, if one presents a data user with a map with predictions made on 100-m blocks, 73 it is reasonable for the user to infer that one is making a tacit claim to be able to identify 74 differences between those blocks, and that features of variation that can be resolved should 75 be assumed to be genuine.

76 Second, while it is true, as Heuvelink (2014) points out, that one can create sets of 77 geostatistical maps in which precision and resolution are decoupled, other things being equal 78 (the sampling grid, the covariates), decreasing the block size reduces the precision of kriged 79 predictions as measured by their mean-square error. Heuvelink (2014) created his sets of 
${ }_{80}$ precise or imprecise and coarse- or fine-resolution maps by working from sparse or dense

${ }_{81}$ data sets. In practice one cannot reduce the grid cell size while maintaining the precision of

82 predictions unless more data or covariates are used for prediction.

83

Third, one may state to the data user that the block size is selected on purely op-

84 erational grounds, and that they should examine the corresponding uncertainty map to

85 understand the claims made for the information. However, while uncertainty measures such

${ }_{86}$ as a kriging variance have considerable value, and are understood by statisticians and sci-

87 entists, it is not clear that they always succeed in conveying to the general data user a clear

88 understanding of uncertainty. Even if one changes the kriging variance to a standard error,

89 so that it is on the scale of the original measurement, the user, particularly one who is

90 interested in the overall variation across a region rather than decision making at one or a

${ }_{91}$ few locations, may be challenged to make a judgement as to which features of the mapped

${ }_{92}$ pattern can be interpreted with confidence and which cannot.

93 In this paper I propose a new measure of the uncertainty of block kriging predictions.

94 This is the block correlation, the expected correlation of the block prediction with the value

95 that it estimates: the spatial mean of the target variable across the block. The block

96 concordance correlation (after Lin, 1989) can also be calculated, and may be more useful for

97 communicating uncertainty of kriged predictions. This is because it measures not simply the

98 linear correlation between variables but their conformity to the 1:1 line. In the case of the

99 correlation between the block spatial mean and the kriging predictor systematic deviations

100 from the 1:1 line are due to over-smoothing by kriging, as the procedure is unbiased.

The block correlation has two particular advantages over kriging variance, which make it pertinent to the questions above. First, it can be compared between blocks of different sizes

${ }_{103}$ more readily than the prediction error variance, because changing block size also changes

104 the variance of the block means. This makes the block correlation conceptually useful

105 for investigating the effects of block size on prediction uncertainty. Second, as a bounded and dimensionless quantity, correlations may be more readily interpreted by the general 
107

user as a measure of information quality than a kriging variance, although this remains a key uncertainty measure for quantitative assessments of uncertainty either through error propagation or the comparison of prediction distributions to threshold values. For reasons explained in the previous paragraph, the block concordance correlation is particularly useful for communication. The user can understand that a concordance correlation of 1 implies that the block prediction is perfect, and zero that the prediction includes no information, with intermediate values implying some degree of deviation from the 1:1 line.

In this paper I develop the concept of the block correlation and concordance correlation and show how they can be computed for block kriging. I then use them to examine the extent to which, ceteris paribus, the block length for the kriged map serves as a proxy for information quality. In particular I consider how the block concordance correlation might be used to select both block size and sample density for a soil map where there is some freedom to select a block size. In principle the block correlation could be fixed at some value as a quality standard and block size adjusted so as to achieve this.

\section{Theory}

\subsection{Block correlation}

Supports are defined in two dimensions, assuming sampling to some fixed depth for intensive properties such as concentrations. The region of interest is denoted by $\mathcal{R} \in \mathbb{R}^{2}$. The variable of interest, measured at location $\mathbf{x} \in \mathcal{R}$, is denoted by $z(\mathbf{x})$ and is treated as a realization of a random variable $Z(\mathbf{x})$. I denote some block of support $\mathcal{B}$ centred at location $\mathbf{x}$ by $\mathbf{x}_{\mathcal{B}}$; so, for example, if $\mathcal{B}$ is a square support of $200 \mathrm{~m} \times 200 \mathrm{~m}$ then $\mathbf{s} \in \mathbf{x}_{\mathcal{B}} \rightarrow \max _{i=1,2}\left|s_{i}-x_{i}\right| \leq 100$. The quantity of interest is the spatial mean of $z$ across ${ }_{29} \mathbf{X}_{\mathcal{B}}$,

$$
\bar{z}\left(\mathbf{x}_{\mathcal{B}}\right)=\int_{\mathbf{s} \in \mathbf{x}_{\mathcal{B}}} z(\mathbf{s}) \mathrm{d} \mathbf{s} .
$$

The ordinary kriging estimate of this quantity is denoted by $\tilde{Z}\left(\mathbf{x}_{\mathcal{B}}\right)$, and the ordinary 
131 kriging variance, $\sigma_{\mathrm{K}}^{2}\left(\mathbf{x}_{\mathcal{B}}\right)$ is obtained with the estimate where

$$
\sigma_{\mathrm{K}}^{2}\left(\mathbf{x}_{\mathcal{B}}\right)=\mathrm{E}\left[\left\{\tilde{Z}\left(\mathbf{x}_{\mathcal{B}}\right)-\bar{z}\left(\mathbf{x}_{\mathcal{B}}\right)\right\}^{2}\right]
$$

By the block correlation we mean

$$
\rho_{\mathcal{B}}=\operatorname{Corr}\left[\tilde{Z}\left(\mathbf{x}_{\mathcal{B}}\right), \bar{z}\left(\mathbf{x}_{\mathcal{B}}\right)\right]
$$

${ }_{133}$ where $\operatorname{Corr}[\cdot, \cdot]$ denotes the correlation of the two variables in the brackets. Note that 134 this quantity is defined for a particular block, given the configuration of sampling points 135 from which the prediction is derived, over the random model which underlies the kriging prediction. It is in this sense a superpopulation statistic, and a measure of confidence for 137 the particular prediction. It should not be confused with a population statistic, such as 138 the correlation of the spatial means of a set of distinct block with their respective kriging 139 predictions from a particular set of data. Bishop et al. (2015) in a recent study attempted to estimate population validation statistics for spatial prediction of soil properties on blocks 141 of different size.

The block correlation can be computed directly from terms computed to solve the ordinary block kriging equation. Because the ordinary kriging predictor is unbiased the 144 ordinary kriging variance is the variance of the difference between the ordinary kriging 145 predictor, $\tilde{Z}\left(\mathbf{x}_{\mathcal{B}}\right)$, and the true spatial mean of the block which it predicts, $\bar{z}\left(\mathbf{x}_{\mathcal{B}}\right)$, and therefore

$$
\operatorname{Cov}\left[\tilde{Z}\left(\mathbf{x}_{\mathcal{B}}\right), \bar{z}\left(\mathbf{x}_{\mathcal{B}}\right)\right]=\frac{\operatorname{Var}\left[\tilde{Z}\left(\mathbf{x}_{\mathcal{B}}\right)\right]+\operatorname{Var}\left[\bar{z}\left(\mathbf{x}_{\mathcal{B}}\right)\right]-\sigma_{\mathrm{K}}^{2}\left(\mathbf{x}_{\mathcal{B}}\right)}{2}
$$

147 where $\operatorname{Cov}[\cdot, \cdot]$ denotes the covariance of the two variables in the brackets and $\operatorname{Var}[\cdot]$ denotes 148 the variance of a variable. The block kriging prediction is obtained from a set of $N$ observations, and the covariance matrix of these observations under the model used for kriging is denoted by $\mathbf{C}$, which 151 is a submatrix of a matrix in the ordinary kriging equation. If $\boldsymbol{\lambda}$ is the vector of ordinary 152 block kriging weights then it follows from the familiar properties of linear combinations of 
153 random variables that

$$
\operatorname{Var}\left[\tilde{Z}\left(\mathbf{x}_{\mathcal{B}}\right)\right]=\boldsymbol{\lambda}^{\mathrm{T}} \mathbf{C} \boldsymbol{\lambda}
$$

154

155

156

${ }_{157}$ variance of $Z$ within the block of support $\mathcal{B}$ defined as

$$
\sigma_{\mathcal{D}, \mathcal{B}}^{2}=\int_{\mathbf{s}_{1} \in \mathbf{x}_{\mathcal{B}}} \int_{\mathbf{s}_{2} \in \mathbf{x}_{\mathcal{B}}} \gamma\left(\mathbf{s}_{1}-\mathbf{s}_{2}\right) \mathrm{d} \mathbf{s}_{2} \mathrm{~d} \mathbf{s}_{1},
$$

158 162 obtain the block correlation

163

$$
\rho_{\mathcal{B}}=\frac{\operatorname{Cov}\left[\tilde{Z}\left(\mathbf{x}_{\mathcal{B}}\right), \bar{z}\left(\mathbf{x}_{\mathcal{B}}\right)\right]}{\sqrt{\operatorname{Var}\left[\tilde{Z}\left(\mathbf{x}_{\mathcal{B}}\right)\right] \operatorname{Var}\left[\bar{z}\left(\mathbf{x}_{\mathcal{B}}\right)\right]}}
$$

where $\gamma(\mathbf{h})$ is the variogram function used for kriging. Again, this term is calculated as part of the computation for ordinary block kriging.

It is therefore possible to calculate all the terms required to find the covariance of the block mean with the ordinary kriging predictor, Eq[4], and this can then be standardized to

The correlation is a measure of the strength of the linear relationship between two variables, and takes values in the interval $[-1,1]$. Because the variances and covariance in Eq [8] are derived from a common stochastic model with authorized (negative semi-definite) variograms, it follows that $\rho_{\mathcal{B}} \in[-1,1]$ for arbitrary real-valued weights in $\boldsymbol{\lambda}$. When $\boldsymbol{\lambda}$ are kriging weights the worse-case scenario for spatial prediction is where $Z$ is a pure nugget random variable with no spatial correlation, and in this case $\rho_{\mathcal{B}}=0$ (see appendix).

One disadvantage of the correlation as a measure of the reproduction of some quantity by a predictor is that it is simply a measure of strength of linear association, and so may take large values even when the predictions are biased, or over- or under-estimate the variance. This was recognized by Lin (1989) who developed the concordance correlation as an alternative. The concordance correlation measures the extent to which a variable and its 
174

associated predictor fall near the 1:1 line. If predictions are perfect (all observations on the 1:1 line) the concordance correlation (and correlation) are 1, if the variable and predictions are independent the expected concordance correlation (and correlation) are zero. However, the predictions and variable may be strongly linearly correlated, but differ markedly with respect to mean, variance or both. In this case the concordance correlation is smaller than the correlation, and a more useful measure of association. The concordance correlation has been used elsewhere in soil science (e.g Corstanje et al., 2008).

The concordance correlation for two variables can be written as

$$
\rho_{\mathrm{c}}=\frac{2 \operatorname{Cov}\left[x, x^{\prime}\right]}{\operatorname{Var}[x]+\operatorname{Var}\left[x^{\prime}\right]+\left\{\mathrm{E}[x]-\mathrm{E}\left[x^{\prime}\right]\right\}^{2}} .
$$

Because ordinary kriging is unbiased the difference between the means in the denominator goes to zero, and so we can write the block concordance correlation as

$$
\rho_{\mathcal{B}, \mathrm{c}}=\frac{\operatorname{Cov}\left[\tilde{Z}\left(\mathbf{x}_{\mathcal{B}}\right), \bar{z}\left(\mathbf{x}_{\mathcal{B}}\right)\right]}{\operatorname{Var}\left[\tilde{Z}\left(\mathbf{x}_{\mathcal{B}}\right)\right]+\operatorname{Var}\left[\bar{z}\left(\mathbf{x}_{\mathcal{B}}\right)\right]} .
$$

Substituting the expression for the covariance in $\mathrm{Eq}[4]$ gives

$$
\rho_{\mathcal{B}, \mathrm{c}}=1-\frac{\sigma_{\mathrm{K}}^{2}\left(\mathbf{x}_{\mathcal{B}}\right)}{\operatorname{Var}\left[\tilde{Z}\left(\mathbf{x}_{\mathcal{B}}\right)\right]+\operatorname{Var}\left[\bar{z}\left(\mathbf{x}_{\mathcal{B}}\right)\right]} .
$$

From Eqs [8] and [10] it can be seen that

$$
\rho_{\mathcal{B}, \mathrm{c}}=2 \rho_{\mathcal{B}} \frac{\sqrt{\operatorname{Var}\left[\tilde{Z}\left(\mathbf{x}_{\mathcal{B}}\right)\right] \operatorname{Var}\left[\bar{z}\left(\mathbf{x}_{\mathcal{B}}\right)\right]}}{\operatorname{Var}\left[\tilde{Z}\left(\mathbf{x}_{\mathcal{B}}\right)\right]+\operatorname{Var}\left[\bar{z}\left(\mathbf{x}_{\mathcal{B}}\right)\right]},
$$

from which it follows that the two correlations are identical if and only if $\operatorname{Var}\left[\tilde{Z}\left(\mathbf{x}_{\mathcal{B}}\right)\right]=$ $\operatorname{Var}\left[\bar{z}\left(\mathbf{x}_{\mathcal{B}}\right)\right]$, and that otherwise $\rho_{\mathcal{B}, \mathrm{c}}<\rho_{\mathcal{B}}$. If the block concordance correlation is smaller than the block correlation this is due to differences in the variance because of the smoothing effect of the kriging predictor, which will be most pronounced when observations are collected on sampling grids which are coarse relative to the range of spatial correlation.

\subsection{Hypothetical examples}

The expressions given above were used to compute block correlations and concordance correlations in different settings. In each case I considered the block correlation and concordance correlation for a cell-centred square block using the nearest 400 observations from a 
square sampling grid. Note that in this paper 'grid spacing' always denotes the spacing of the sample grid. Grid spacings from 100 to $1000 \mathrm{~m}$ were considered, and correlations were computed for square blocks of length $10 \mathrm{~m}$ to $500 \mathrm{~m}$.

Three spatial models were considered. In each case there was a nugget component with variance $c_{0}$, and two nested spherical components one of range $250 \mathrm{~m}$ (comparable to the shorter grid spacings considered) and variance $c_{1}$ and one of range $5000 \mathrm{~m}$ (longer than any grid spacings) and variance $c_{2}$. The first model was 'nugget-dominated' with $c_{0}=0.7, c_{1}=0.1, c_{2}=0.2$. The second model was dominated by the short range term: $c_{0}=0.1, c_{1}=0.7, c_{2}=0.2$. The third model was long-range-dominated: $c_{0}=0.1, c_{1}=$ $0.2, c_{2}=0.7$. Figure 1 shows the block correlations and block concordance correlations for each model plotted as contours to show the effect of grid spacing and block length, and Figure 2 shows block correlation and block concordance correlation plotted against block length for a 300-m sampling grid.

The following key properties of the block correlation emerge

i. For any grid spacing the block correlation increases with block length, but the effect of block length depends on the dominant scales of variation relative to block size and grid spacing. In the short-range-dominated case the block correlation increases more rapidly with block length with smaller grid spacings than with coarser ones. In the long-range-dominated case the block correlation is insensitive to block length, other factors remaining constant.

ii. For any block size the block correlation declines with increasing grid spacing. The sensitivity to grid spacing depends on the correlation structure of the random variable, and on the grid spacing in the short-range-dominated case.

iii. For any fixed grid spacing and block size the block correlation is smallest in the shortrange dominated case and largest in the long-range dominated case.

The first point shows that the effect of block length on uncertainty of the prediction depends strongly on the important scales of spatial variation and the sampling intensity. 
${ }_{222}$ The effect may be small, in which case there is no strong reason to take uncertainty and its 223 communication into account when selecting a block length. However, in other conditions (as 224 in the short-range dominated case), with sampling on a grid of spacing similar in order to the range of correlation of a component of the variable, the uncertainty of a block prediction 226 may be very sensitive to block size. Note, in respect of point (iii) that the variance at lags greater than $250 \mathrm{~m}$ is the same

in the short-range and nugget-dominated cases. When most of this variance is in the nugget term then the effect of this on the uncertainty of the block mean is removed by any spatial aggregation, and the block size has little effect. It is the short-range component of variation which largely contributes to the increase in block correlation with block length. There is a practical consequence of this effect. Consider a case in which all information on a variable with the short-range dominated variability was available from a 400-m grid. In this case the short-range component would not be resolved, and its variance would all be attributed to a nugget component. This would result in overestimation of the block correlation. It is important to base sampling decisions on variograms estimated from sampling schemes that provide information on short lags relative to potential block sizes. However, a conservative approach would be to compute block correlations with a variogram function in which the nugget component is replaced with a nested spherical or other authorised model with variance equal to the nugget and range equal to the shortest lag distance at which the variogram is estimated from supporting data. Note that the model substituted for the lag should be one like the spherical where the autocorrelation goes exactly to zero at the lag. If the measurement error of the variable is known independently then the nugget variance could be partitioned into a measurement error component and a component of variance correlated at fine scales. Only the latter component would be treated as correlated up to the shortest lag interval in the data, and the measurement error component treated as a nugget effect.

The block concordance correlations in Figure 1 show similar behaviour to the block correlations, with similar effects of grid size and block length depending on the underlying 
249

spatial dependence. In Figure 2 there is little difference between the block correlations and block concordance correlations for different block lengths with a sample grid spacing of 300-m in the long-range-dominated and nugget-dominated cases. However, the block concordance correlation decreases more markedly with block length than does block correlation in the short-range-dominated case.

Figure 3 shows the ratio of block concordance correlation to block correlation for different block lengths and grid spacings in the short-range-dominated, nugget-dominated and long-range-dominated cases. In the latter two cases the ratio is nowhere very far from 1.0, but in the short-range dominated case the minimum value over the cases explored is a little less than 0.6 . The ratio is reduced by predicting means for smaller blocks or by increasing the grid spacing. This indicates that the tendency for the kriging predictor to smooth is increased by sparser sampling but also by predicting for smaller blocks. This latter effect is only made clear by the block concordance correlation. It would not be apparent in a visual assessment of block kriging on different supports because the absolute variance of the smaller blocks is larger.

The block concordance correlation shows comparable effects of block length on prediction uncertainty to the block correlation. It is more useful than the block correlation as an absolute measure of uncertainty because it can be understood as a measure of scatter about the 1:1 line and not just a measure of linear association. I therefore suggest that the block concordance correlation is preferred for communication of the uncertainty of block kriging predictions, and the selection of a block size where there is flexibility to adjust this.

\section{A case study with soil data}

Next I present block correlations computed from a variogram for a soil property. The property is total nickel $(\mathrm{Ni})$ content of topsoil determined from soil samples collected across the Humber-Trent region of eastern England as part of the Geochemical Baselines Survey of the Environment (Rawlins et al., 2003; Johnson et al., 2005). The data were obtained from sample sites at a mean density of about one per $2 \mathrm{~km}^{2}$ across the region. Each sample 
276

was a composite formed from cores collected at the centre and vertices of a 20 -m square.

The cores were length $15 \mathrm{~cm}$ excluding surface litter. Material was subsequently air-dried, disaggregated and sieved to pass $2 \mathrm{~mm}$ and sub-sampled by coning and quartering. A 50-g sub-sample was ground in an agate planetary ball mill until $95 \%$ of the material was finer than $53 \mu \mathrm{m}$. Total concentration of $\mathrm{Ni}$, along with 25 other elements was determined for each sample by wavelength dispersive X-Ray Fluorescence Spectrometry.

Details of the analysis of these data are provided by Lark and Lapworth (2013). In summary, exploratory analysis suggested that there was no pronounced anisotropy in this variable. Isotropic variograms were estimated using the standard estimator of Matheron (1962) and alternative estimators, including the resistant estimator of Cressie and Hawkins (1980). Models were fitted to each set of estimates by weighted least squares and then tested by cross-validation. For each model the standardized squared cross-validation error, the square error divided by the point ordinary kriging variance, was computed for each datum and, following Lark (2000) the median value over all data was computed as a validation statistic. Lark and Lapworth (2013) tabulate the validation statistics. On the basis of these the model fitted to the empirical variogram obtained with the estimator of Cressie and Hawkins (1980) was selected. The estimates and fitted model are shown in Figure 4. Note that the model was fitted to variogram estimates at lag distances from approximately $200 \mathrm{~m}$ to $30000 \mathrm{~m}$. The model is a double spherical with distance parameters of 2535 and $16115 \mathrm{~m}$ and corresponding variance components of 42.5 and 82.7 respectively. The nugget variance is 11.6 .

The procedures described above were used to compute block correlations and block concordance correlations for $\mathrm{Ni}$ on square blocks from $10 \mathrm{~m}$ to $1 \mathrm{~km}$ in length from square grids of interval 2 to $5 \mathrm{~km}$. Note that, as discussed in section 2.2 , the block correlations were computed with a variogram model identical to the fitted one shown in Figure 4 except that the nugget component was replaced by a spherical variogram with range $200 \mathrm{~m}$ and variance equal to the nugget variance in the fitted model. Figure 5 shows contours of the 
303

block correlation and concordance correlation over the range of block sizes and grid spacings considered. Over this space the block correlation varies from 0.60 to 0.86 and the block concordance correlation varies from 0.55 to 0.85 . Figure 6 shows graphs of block correlation and concordance correlation against block length for $2-\mathrm{km}$ and 5 -km sample grids. With a 2-km square grid, the average sampling intensity of the Humber-Trent survey, the block concordance correlation is 0.8 for a $350-\mathrm{m}$ block. This block length could be selected for kriging the variable to provide an overview of variation in nickel concentration when other operational factors do not determine the block size. However, the contour map for block correlations in Figure 5 shows that the sensitivity of the block correlation to block length is not very great for fixed grid spacing over the range considered. For example, if one preferred to use a 100-m block for practical reasons for prediction from a 2-km sampling grid the block concordance correlation is 0.76 . This is because most of the variance $(60 \%)$ is spatially correlated with a range of $2.5-\mathrm{km}$ or more. With a 5 -km sample grid — the sample intensity of the National Soil Inventory in England and Wales, (McGrath and Loveland, 1992) — the block concordance correlation for a $350-\mathrm{m}$ block is 0.58 . As seen in Figures 5 and 6 , the block concordance correlation is not very sensitive to block length, and increasing the block length to $1000 \mathrm{~m}$ increases it to just 0.63 . The dominant limitation on the confidence we can have in kriged results is the sampling density rather than the block size. For comparison

Figure 7 shows block kriging variances for blocks up to $1000 \mathrm{~m}$ in length for prediction from 2-km and 5-km sampling grids.

Figure 8 shows block-kriged maps of Ni across the region with square blocks of 350-m length, and the local kriging variance, and Figure 9 shows the block correlations and block concordance correlations computed for each block over the stochastic model. Note that the concordance correlations are mostly larger than 0.8 (the median value is 0.88 ), which is the block correlation for a worst-case scenario prediction from a 2-km square grid: a cell-centred block as far as possible from any observations. The kriging variances, block correlations and block concordance correlations show some variation, since the sampling intensity is smaller 
330

\section{Discussion}

The key finding of this paper is that one cannot generalize about the relationship between block size and prediction quality. While increasing the block size increases block correlation and concordance correlation, the effect may be very small in cases where the variation is dominated by a component with a range of correlation which is long relative to grid spacing and block size. Thus, as in the Humber-Trent case, reducing the block length from $350 \mathrm{~m}$ to $100 \mathrm{~m}$ reduces block concordance correlation by a small amount $(0.80$ to 0.76$)$, particularly if one considers the inevitable uncertainty in the variogram estimate. The block length has no bearing on uncertainty of the predictions and can be selected on other criteria. If the block correlation is not deemed sufficient then this can be improved only by increasing the density of sampling, or finding an appropriate covariate.

However, in cases where the sample grid spacing is of similar order to the correlation range of a significant component of the random variable, block correlation can be sensitive to block length. In the short-range-dominated hypothetical example (Figures 1 and 2) with a sample grid of $300 \mathrm{~m}$, the block concordance correlation is increased from 0.35 to 0.80 by using a $350-\mathrm{m}$ block rather than a 50-m block. In these circumstances careful attention should be paid to both sample spacing and block length, and it could be concluded that making predictions on a 50-m block is not justified without increasing the sample density. More generally one might adhere to an operational rule that, at least when mapping to provide a synoptic overview, the block length is selected to achieve a block concordance correlation of no less than 0.8 , and if this is not achievable at the sample density available then this is flagged for the data user. If the blocks were fixed at $50 \mathrm{~m}$ for practical reasons (management zones, for example), then the data user should be aware of the weak block concordance correlations and encouraged to examine the kriging variances and assess the implications of this uncertainty for any decisions made with the data.

In addition to these observations it is suggested that the block concordance correla- 
${ }_{357}$ tion is used as an alternative to the kriging variance as the primary means to communicate the uncertainty of geostatistical maps, particularly for general users interested in a synoptic overview of the soil variable rather than those making specific decisions from the predictions 360 (e.g. on land remediation) where more focussed decision analysis is necessary. In partic361 ular one might use a verbal scale to indicate the strength of correlation such as the one 362 proposed by Campbell and Swinscow (2011) whereby a correlation in the interval 0-0.19 is 363 called 'very weak', $0.2-0.39$ is 'weak', $0.40-0.59$ is 'moderate', $0.6-0.79$ is 'strong' and $>0.8$ 364 is 'very strong'. It would be advisable to include the numerical values of the concordance 365 correlation along with the verbal labels to improve the consistency of these interpretations 366 by data users, and to avoid regressive interpretations (Budescu et al., 2009). If the kriging 367 variances are also provided then the user, having formed an initial impression of uncertainty from the concordance correlations may be able to consider the implications of the confidence intervals of the kriging predictions on the scale of measurement of the target variable. This approach is consistent with the recommended practice of 'progressive disclosure' of informa371 tion about uncertainty (e.g. Wardekker et al., 2008). More accessible and general measures 372 of uncertainty are provided initially as a prelude to more focussed measures, which may require greater statistical understanding and may not be needed by all data users. In this paper I have introduced the block correlation and concordance correlation in 375 the context of ordinary univariate kriging. However, these statistics could be computed 376 in a straightforward way for predictions obtained by other model-based methods. The 377 formulation of the block concordance correlation in Eq [11], for example, shows that we require the prediction error variance, variance of the block spatial mean and variance of the block mean estimate. In the case of block cokriging the first of these terms is the block cokriging variance, and the second two terms can be obtained from equations in this paper ${ }_{381}$ and the autovariogram for the target variable in the linear model of coregionalization. In the ${ }_{382}$ case of a best linear unbiased prediction with one or more covariates (kriging with external 383 drift) the block correlation or concordance correlation is conditional on the block mean of the 
384

\section{Acknowledgements}

The contribution of all British Geological Survey staff involved in the geochemical sampling of the Humber Trent region, and the subsequent soil analysis, is acknowledged. This paper is published with the permission of the Director of the British Geological Survey (NERC). I am grateful for helpful comments by the Editor and two reviewers, one of whom suggested the inclusion of the concordance correlation. 


\section{References}

Arrouays, D., McBratney, A.B., Minasny, B., Hempel, J.W. et al. 2014a. The GlobalSoilMap project specifications. In: Arrouays, D., McKenzie, N., Hempel, J., Richer de Forges, A.C., McBratney, A. (Eds.) GlobalSoilMap, Basis of the global spatial soil information system. CRC Press, Boca Raton. pp, 9-12.

Arrouays, D., Grundy, M.G., Hartemink, A.E., Hempel, J.W., et al. 2014b. GlobalSoilMap: toward a fine-resolution global grid of soil properties. Advances in Agronomy $125,93-134$.

Beckett, P.H.T., Burrough, P.A. 1971. The relation between cost and utility in soil survey. IV. Comparison of the utilities of soil maps produced by different survey procedures, and to different scales. Journal of Soil Science 22, 466-480.

Bishop, T.F.A., Horta, A., Karunaratne, S.B. 2015. Validation of digital soil maps at different spatial supports. Geoderma, 241-242, 238-249.

Budescu, D.V., Broomell, S., Por, H.-H. 2009. Improving communication of uncertainty in the reports of the Intergovernmental Panel on Climate Change. Psychological Science 20, 299-308.

Burrough, P.A., Beckett, P.H.T. 1971. The relation between cost and utility in soil survey. I. The design of the Experiment. Journal of Soil Science 22, 360-368.

Campbell, M.J., Swinscow, T.D.V. 2011. Statistics at square one. John Wiley and Sons, Chichester.

Corstanje, R.,Kirk, G.J.D., Lark, R.M. 2008. The behaviour of soil process models of ammonia volatilization at contrasting spatial scales. European Journal of Soil Science, $59,1271-1283$.

Cressie, N., Hawkins, D. 1980. Robust estimation of the variogram. Journal of the International Association of Mathematical Geology 12, 115-125.

Dent, D., Young, A. 1981. Soil Survey and Land Evaluation. George Allen and Unwin, London.

Gunn, R.H., Beattie, J.A., Riddler, A.M.H., Lawrie, R.A. 1988. Mapping. In: Gunn, R.H., Beattie, J.A., Reid, R.E., van de Graaff, R.H.M. (Eds.), Australian soil and land survey handbook: guidelines for conducting surveys. Inkata Press, Melbourne. 
Hempel, J.W., McBratney, A.B., Arrouays, D., McKenzie, N.J., Hartemink, A.E. 2014. GlobalSoilMap project history. In: Arrouays, D., McKenzie, N., Hempel, J., Richer de Forges, A.C., McBratney, A. (Eds.) GlobalSoilMap, Basis of the global spatial soil information system. CRC Press, Boca Raton. pp, 3-8.

Hengl, T. 2006. Finding the right pixel size. Computers and Geosciences, 32, 1283-1298.

Heuvelink, G.B.M. 2014. It's the uncertainty, stupid. Pedometron, 36, 19-21.

Johnson, C.C., Breward, N., Ander, E. L., Ault, L. 2005. G-BASE: Baseline geochemical mapping of Great Britain and Northern Ireland. Geochemistry: Exploration, Environment, Analysis 5, 1-13.

Journel, A.G., Huijbregts, C.J., 1978, Mining Geostatistics. Academic Press, London.

Lark, R.M. 2000. A comparison of some robust estimators of the variogram for use in soil survey. European Journal of Soil Science 51, 137-157.

Lark, R.M., Lapworth, D.J. 2013. A novel quality measure for planning geochemical surveys of the soil by kriging. Geoderma, 197-198, 27-35.

Lin, L. I-K. 1989. A concordance correlation coefficient to evaluate reproducibility. Biometrics, $45,255-268$.

Matheron, G. 1962. Traité de Géostatistique Appliqué, Tome 1. Mémoires du Bureau de Recherches Géologiques et Minières, Paris.

McGrath, S.P., Loveland, P.J. 1992. The Soil Geochemical Atlas of England and Wales. Blackie, London.

Rawlins, B.G., Webster, R., Lister, T.R. 2003. The influence of parent material on topsoil geochemistry in Eastern England. Earth Surface Processes and Landforms 28, 13891409.

Resources Inventory Committee. 1995. Soil Inventory Methods for British Columbia, British Columbia Ministry of Environment. http://www.for.gov.bc.ca/hts/risc/ pubs/teecolo/soil/soil_inv_methodsbc.pdf

Tobler, W. 1988. Resolution, resampling and all that. In: Mounsey, H., Tomlinson, R.F. (Eds.), Building databases for global science. Taylor and Francis, London. 129-137. 
Wardekker, J.A., van der Sluijs, J.P., Janssen, P.H.M., Kloprogge, P., Petersen, A.C. 2007. Uncertainty communication in environmental assessments: views from the Dutch science-policy interface. Environmental Science \& Policy, 11, 627-641.

Webster, R., Oliver, M.A. 2007. Geostatistics for Environmental Scientists. 2nd Edition John Wiley \& Sons, Chichester. 
Appendix. For ordinary block kriging with a pure nugget variogram, $\rho_{\mathcal{B}}$ and $\rho_{\mathcal{B}, c}$ are zero.

The ordinary block kriging weights for prediction from $n$ unique observation sites, $\lambda_{i}, i=1,2, \ldots n$, are found by solution of the equation:

$$
\mathbf{A} \boldsymbol{\lambda}_{k}=\mathbf{b}
$$

where

$$
\mathbf{b}=\left[\bar{\gamma}_{1}, \bar{\gamma}_{2}, \ldots, \bar{\gamma}_{n}, 1\right]^{\mathrm{T}}
$$

and $\bar{\gamma}_{i}$ denotes the mean semivariance between the $i$ th observation and the block. Matrix $\mathbf{A}$ is $(n+1) \times(n+1)$ with all diagonal elements zero, and off-diagonal elements $[i, j]$ equal to the semivariance between the $i$ th and $j$ th observation if $i \leq n$ and $j \leq n$. All off-diagonal elements in the $(n+1)$ th row and column are 1 . In addition

$$
\boldsymbol{\lambda}_{\mathrm{k}}=\left[\lambda_{1}, \lambda_{2}, \ldots, \lambda_{n}, \psi\right]^{\mathrm{T}},
$$

where $\psi$ is a Lagrange multiplier (Webster and Oliver, 2007). In the case of the pure nugget variogram all observations are uncorrelated with each other and with the block so all weights are equal, and, because of the Lagrange multiplier required by the unbiasedness condition of ordinary kriging,

$$
\lambda_{i}=\frac{1}{n}, \quad i=1,2, \ldots, n
$$

In the case of the pure nugget variogram, all off-diagonal elements of $\mathbf{A}[i, j], i \leq n$ and $j \leq n$ are equal to $\operatorname{Var}[Z]$, as are the first $n$ elements of $\mathbf{b}$. Any of the first $n$ equations in the system in Eq [13] therefore takes the form

$$
\frac{n-1}{n} \operatorname{Var}[Z]+\psi=\operatorname{Var}[Z],
$$

and so

$$
\psi=\frac{\operatorname{Var}[Z]}{n} .
$$

The ordinary block kriging variance is

$$
\sigma_{\mathrm{K}}^{2}\left(\mathbf{x}_{\mathcal{B}}\right)=\mathbf{b}^{\mathrm{T}} \boldsymbol{\lambda}_{\mathrm{k}}-\sigma_{\mathcal{D}, \mathcal{B}}^{2},
$$

(Webster and Oliver, 2007).

We can therefore write from Eqs [4],[6] and [16]

$$
\operatorname{Cov}\left[\tilde{Z}\left(\mathbf{x}_{\mathcal{B}}\right), \bar{z}\left(\mathbf{x}_{\mathcal{B}}\right)\right]=\frac{\operatorname{Var}\left[\tilde{Z}\left(\mathbf{x}_{\mathcal{B}}\right)\right]+\operatorname{Var}[Z]-\mathbf{b}^{\mathrm{T}} \boldsymbol{\lambda}_{\mathrm{k}}}{2} .
$$


In the pure nugget case

$$
\operatorname{Var}\left[\tilde{Z}\left(\mathbf{x}_{\mathcal{B}}\right)\right]=\boldsymbol{\lambda}^{\mathrm{T}} \mathbf{C} \boldsymbol{\lambda}=\frac{\operatorname{Var}[Z]}{n},
$$

as all elements in $\boldsymbol{\lambda}$ are $\frac{1}{n}$ and $\mathbf{C}=\operatorname{diag}\{\operatorname{Var}[Z]\}$; it is also the case that

$$
\mathbf{b}^{\mathrm{T}} \boldsymbol{\lambda}_{\mathbf{k}}=\operatorname{Var}[Z]+\psi
$$

Substituting Eqs [18] and [19] into Eq [17], and noting Eq [15] gives

$$
\begin{aligned}
\operatorname{Cov}\left[\tilde{Z}\left(\mathbf{x}_{\mathcal{B}}\right), \bar{z}\left(\mathbf{x}_{\mathcal{B}}\right)\right] & =\frac{1}{2}\left(\frac{\operatorname{Var}[Z]}{n}-\psi\right) \\
& =0
\end{aligned}
$$

from which it follows that $\rho_{\mathcal{B}}$ and $\rho_{\mathcal{B}, \mathrm{c}}$ are zero 


\section{Figure captions}

1. Block correlation (left) and block concordance correlation (right) as a function of grid spacing and block length for nugget-dominated, short-range-dominated and longrange-dominated random variables.

2. Block correlation as a function of block length for nugget-dominated, short-rangedominated and long-range-dominated random variables kriged from a 300-m square grid.

3. The ratio of block concordance correlation to block correlation as a function of grid spacing and block length for nugget-dominated, short-range-dominated and long-rangedominated random variables.

4. Empirical variogram for topsoil Ni content in the Humber-Trent region with fitted double-spherical model.

5. Block correlation (left) and block concordance correlation (right) as a function of grid spacing and block length for $\mathrm{Ni}$ in the Humber-Trent region.

6. Block correlation (left) and block concordance correlation (right) as a function of block length for $\mathrm{Ni}$ in the Humber-Trent region kriged from a 2-km or 5-km square grid.

7. Block kriging variance as a function of block length for $\mathrm{Ni}$ in the Humber-Trent region kriged from a $2-\mathrm{km}$ or 5 -km square grid.

8. Kriged estimates of $\mathrm{Ni}$ content on discrete $350-\mathrm{m}$ blocks across the Humber-Trent region (Top) and corresponding block kriging variances (Bottom).

9. Block correlations (top) and concordance correlations (bottom) for kriged estimates of Ni content on 350-m blocks across the Humber-Trent region. 


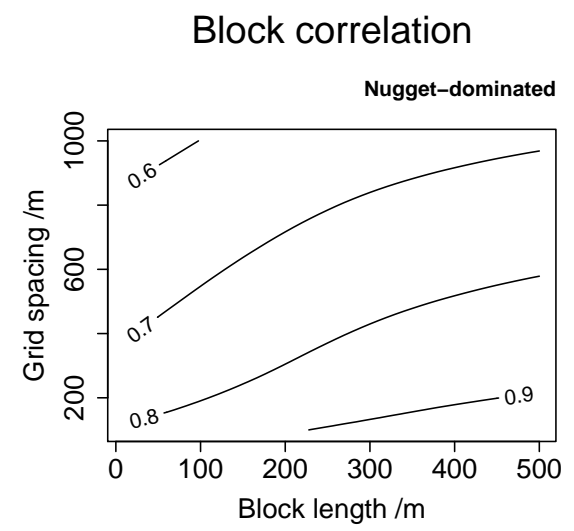

\section{Block concordance correlation}
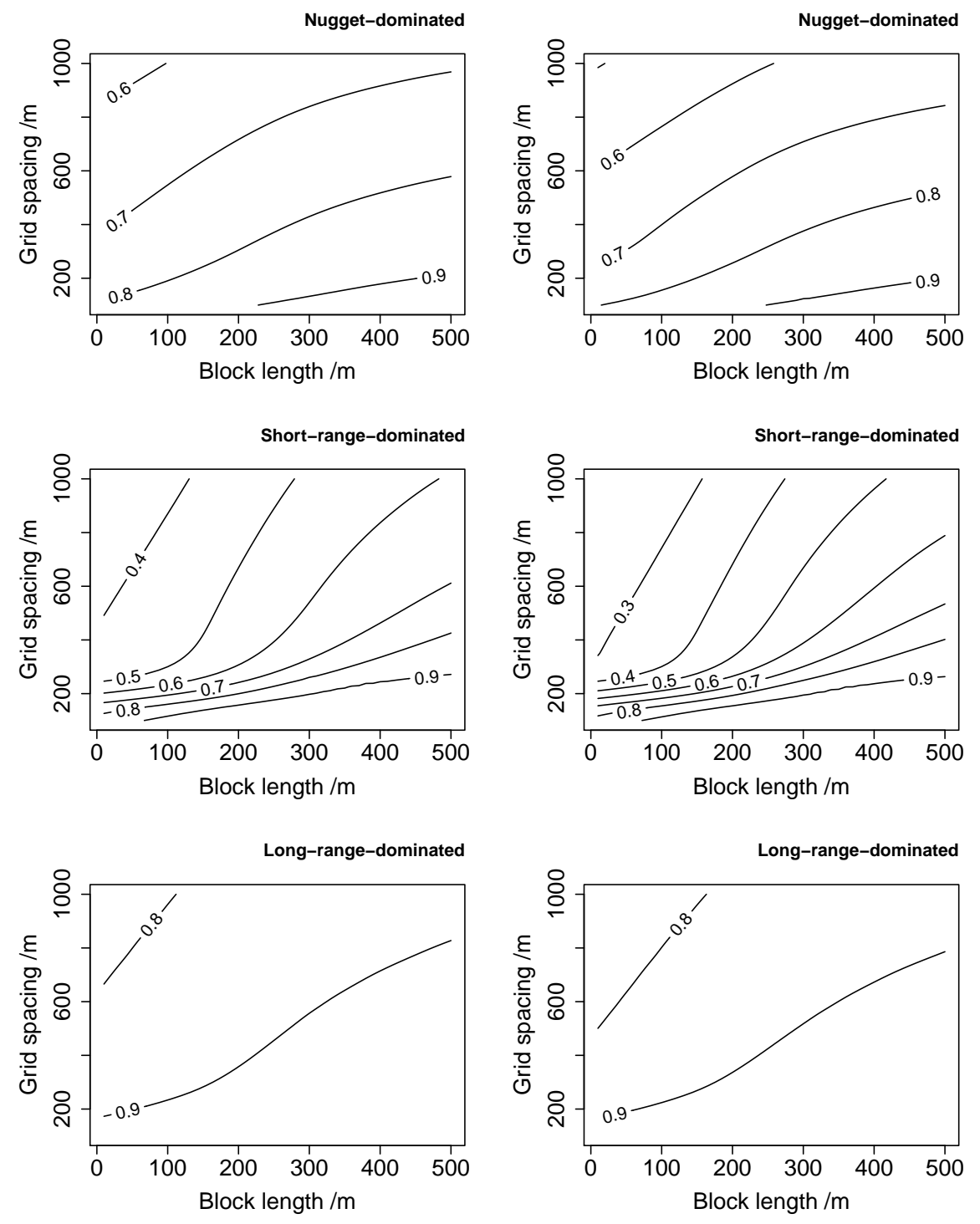

Figure 1: Block correlation (left) and block concordance correlation (right) as a function of grid spacing and block length for nugget-dominated, short-range-dominated and long-rangedominated random variables. 


\section{Block correlation}

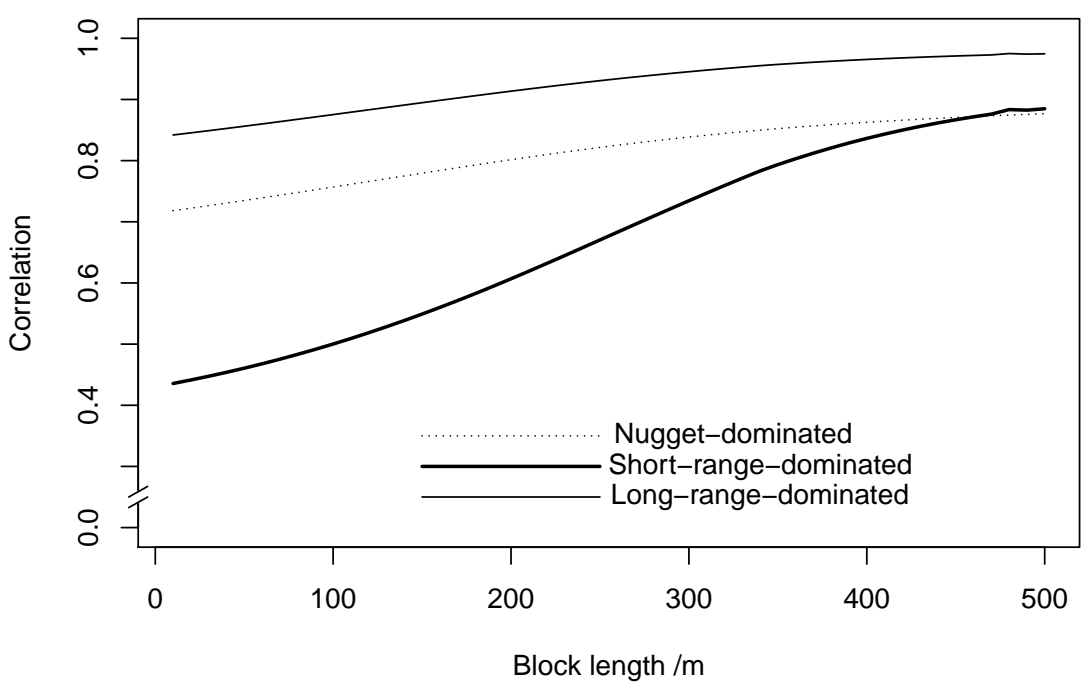

Block concordance correlation

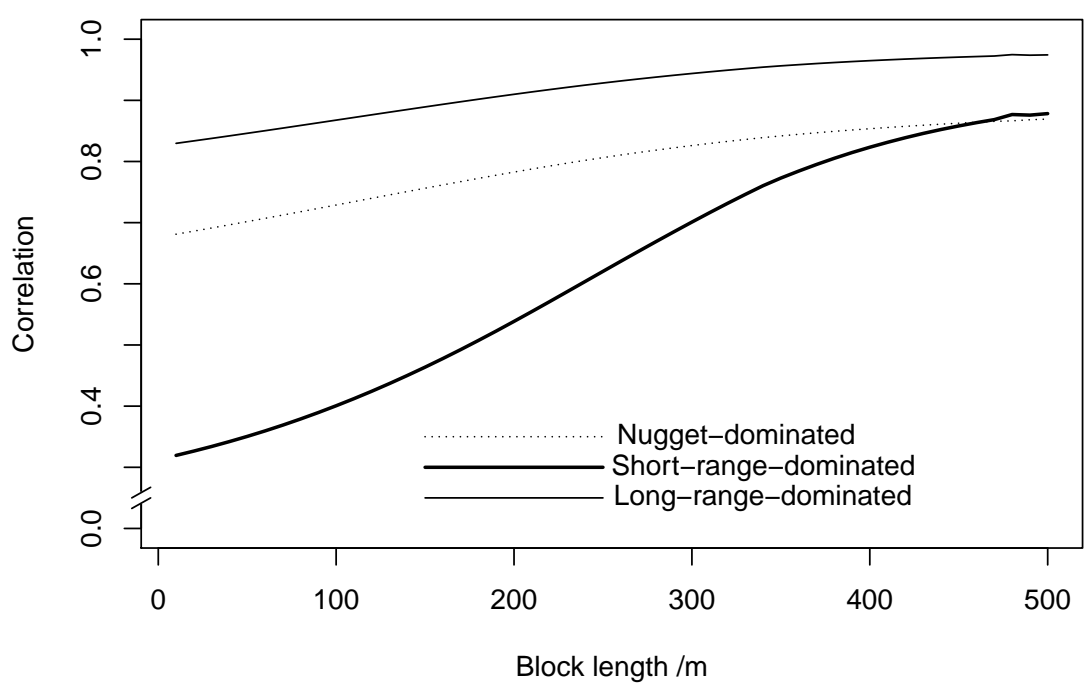

Figure 2: Block correlation as a function of block length for nugget-dominated, short-rangedominated and long-range-dominated random variables kriged from a 300-m square grid. 


\section{Block concordance correlation/Block correlation}
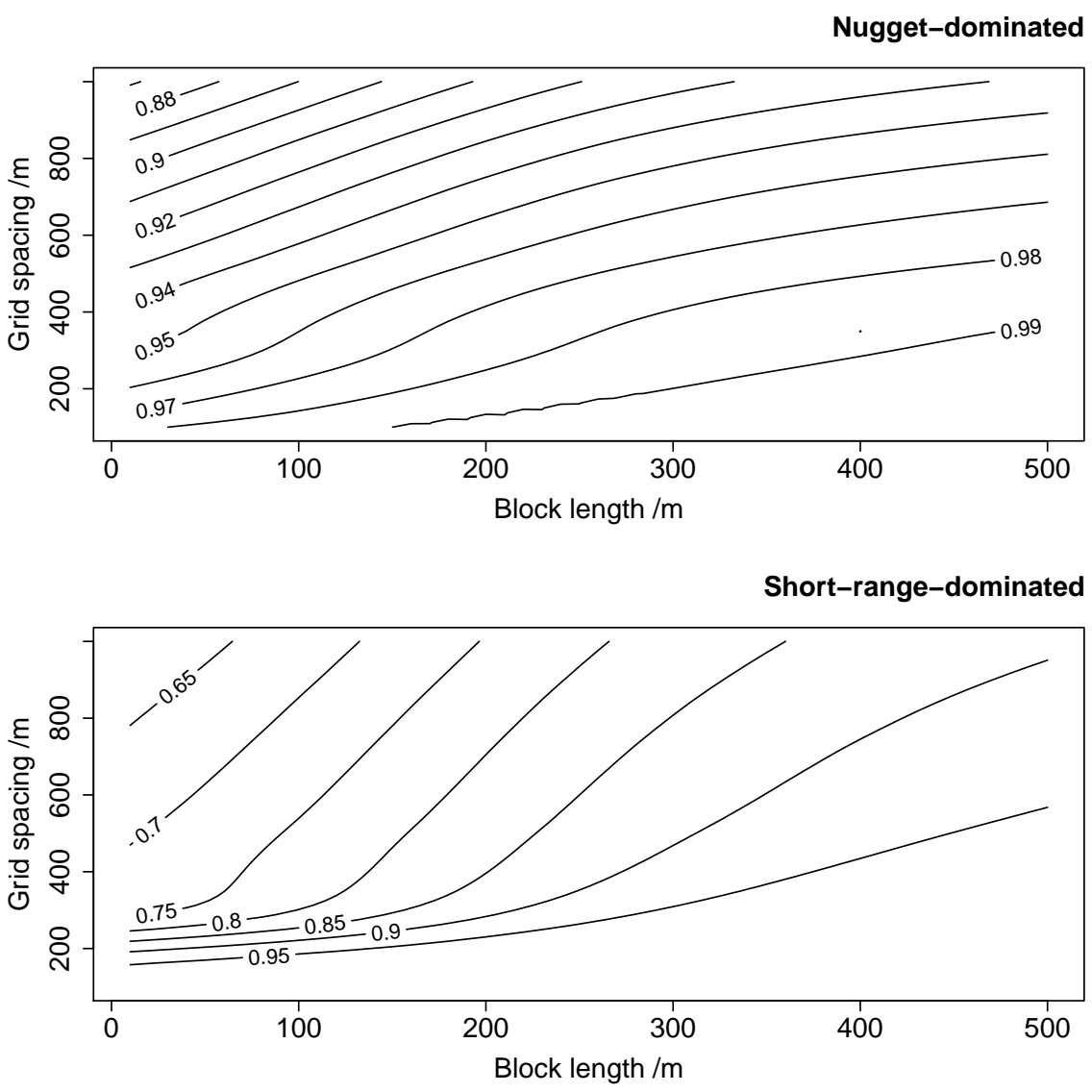

Long-range-dominated

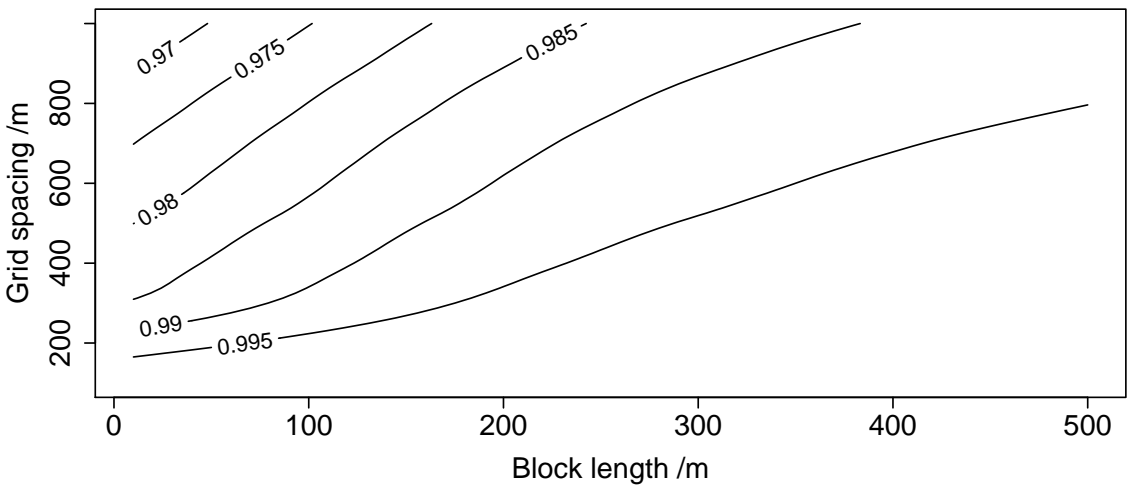

Figure 3: The ratio of block concordance correlation to block correlation as a function of grid spacing and block length for nugget-dominated, short-range-dominated and long-rangedominated random variables. 


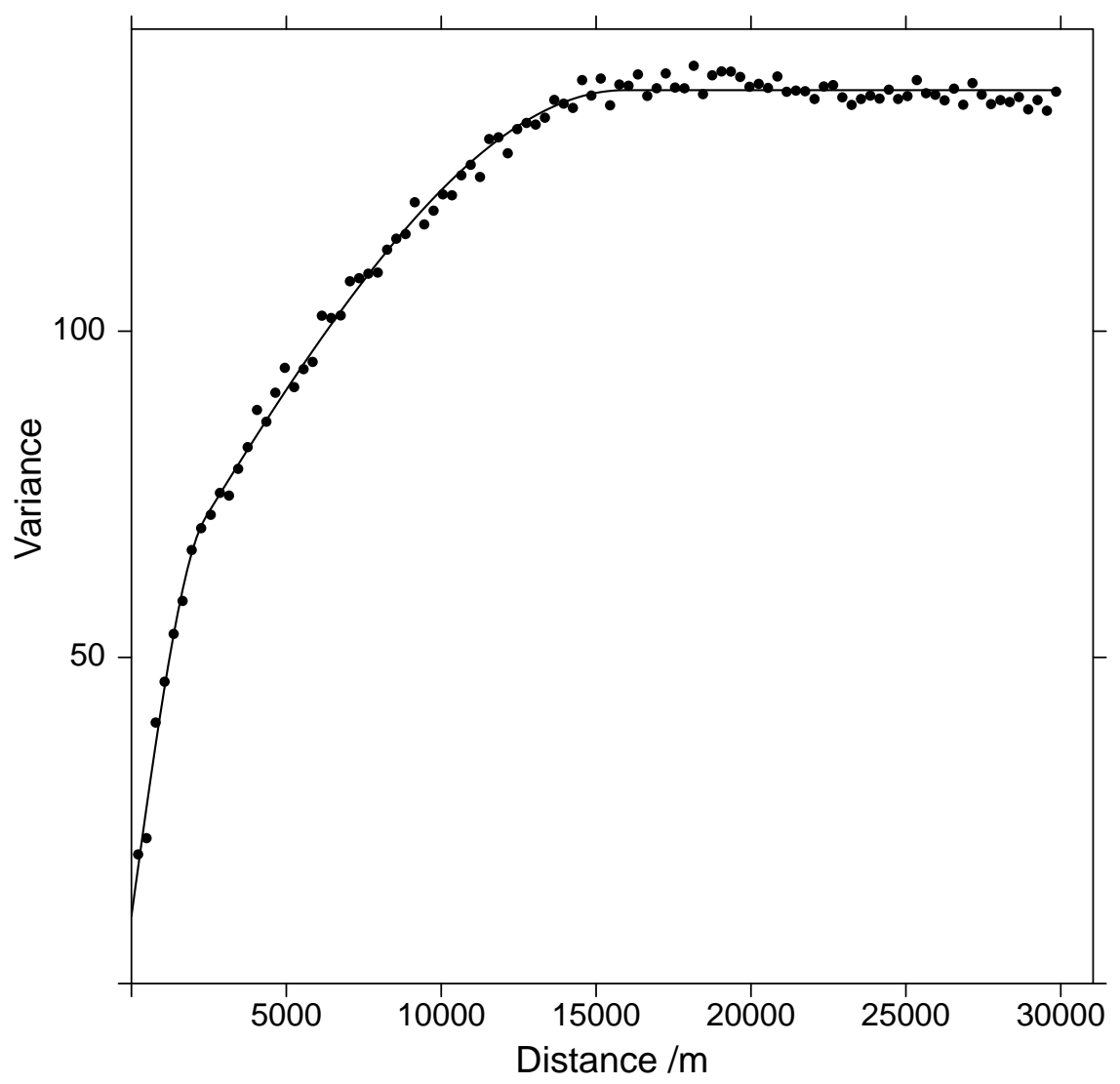

Figure 4: Empirical variogram for topsoil Ni content in the Humber-Trent region with fitted double-spherical model. 


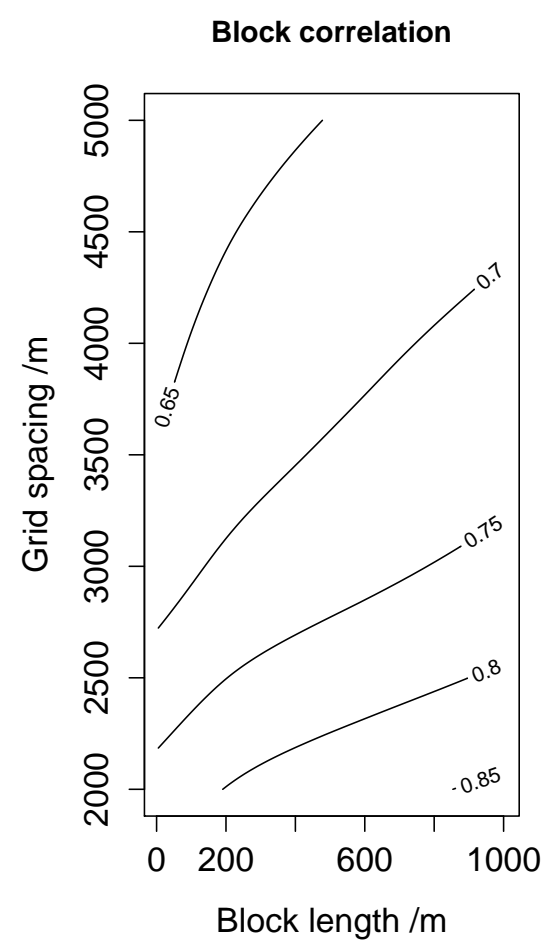

Block concordance correlation

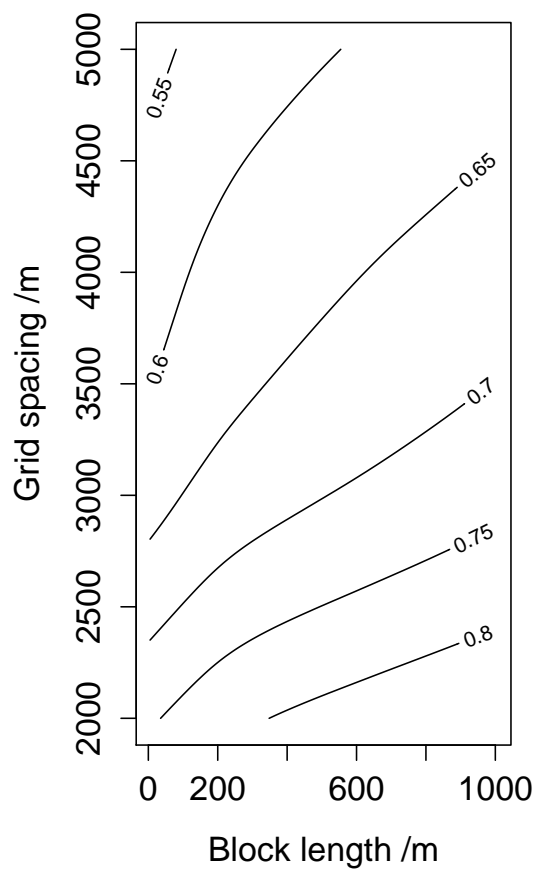

Figure 5: Block correlation (left) and block concordance correlation (right) as a function of grid spacing and block length for $\mathrm{Ni}$ in the Humber-Trent region. 

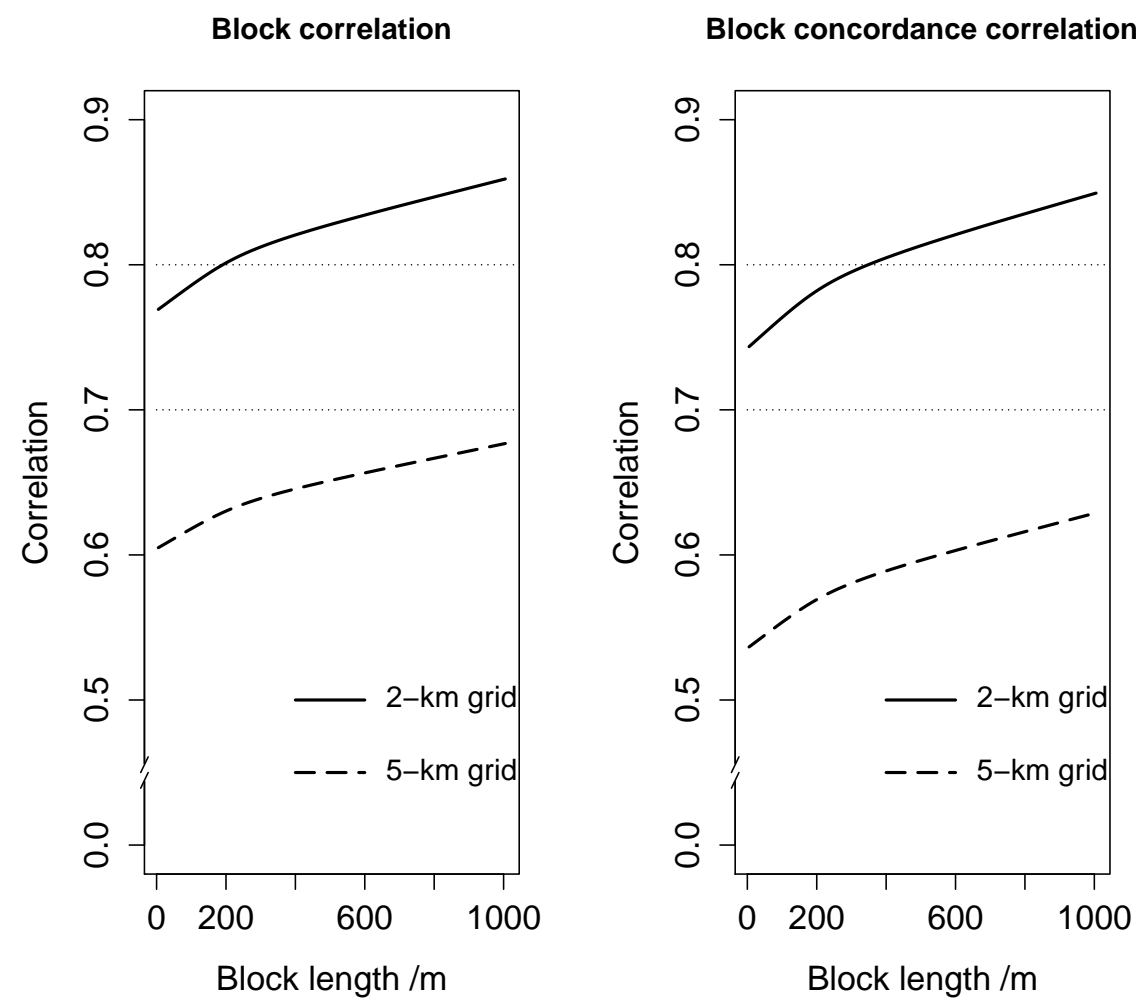

Figure 6: Block correlation (left) and block concordance correlation (right) as a function of block length for $\mathrm{Ni}$ in the Humber-Trent region kriged from a 2-km or 5-km square grid. 


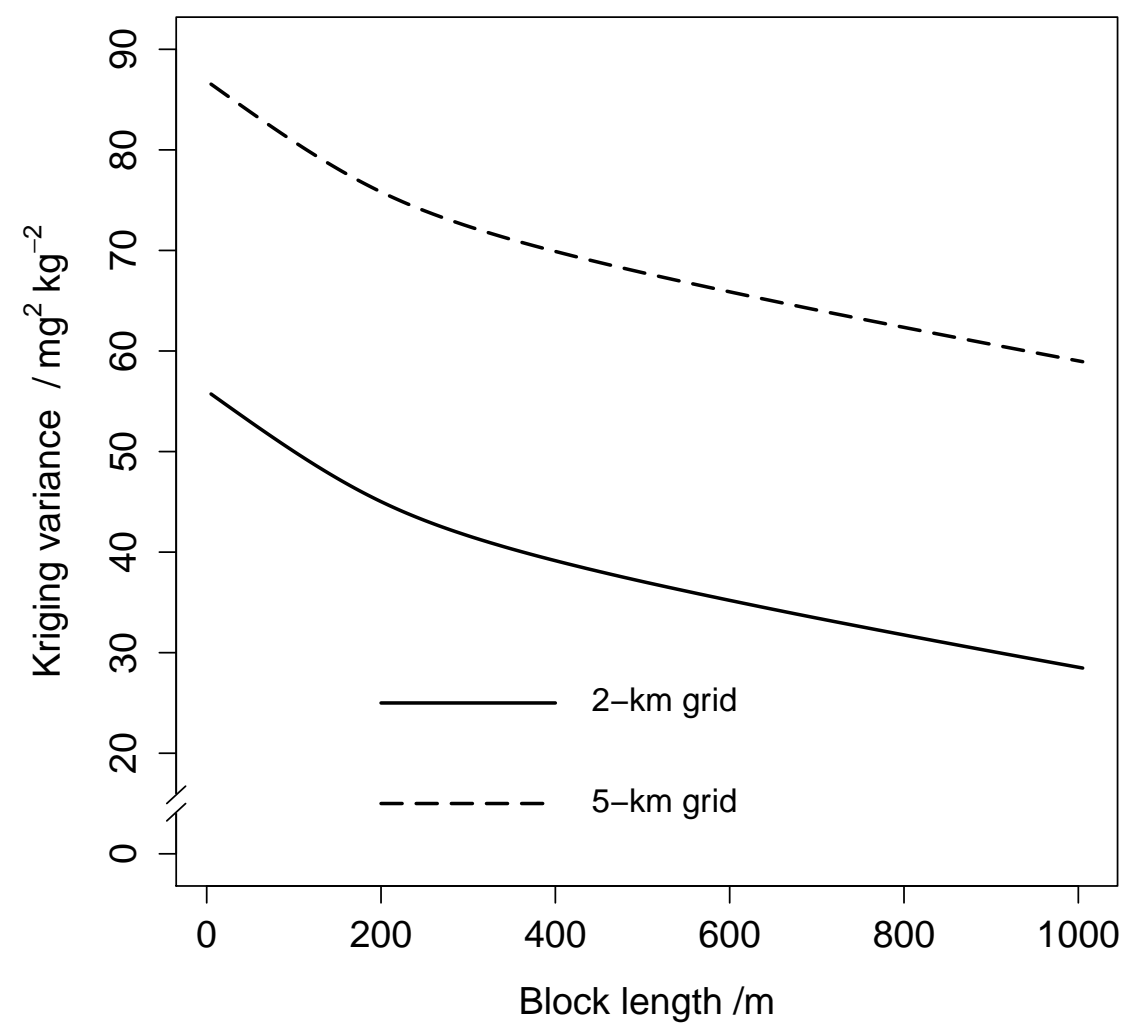

Figure 7: Block kriging variance as a function of block length for Ni in the Humber-Trent region kriged from a $2-\mathrm{km}$ or 5 -km square grid. 
